\title{
Avaliação dos serviços farmacêuticos na Atenção Primária à Saúde no cuidado ao paciente com tuberculose
}

\author{
Evaluation of pharmaceutical services in primary health care in the \\ care of patients with tuberculosis
}

Fernanda de Farias Rodrigues ${ }^{\mathbf{1}}$, Rosana Aquino ${ }^{\mathbf{2}}$, Maria Guadalupe Medina ${ }^{\mathbf{3}}$

DOI: 10.1590/0103-11042018S212

1 Universidade Federal da Bahia (UFBA), Instituto de Saúde Coletiva (ISC) Salvador (BA), Brasil. Orcid: https://orcid. org/0000-0002-99949982

frodrigues.far@gmail.com

2 Universidade Federal da Bahia (UFBA), Instituto de Saúde Coletiva (ISC) Salvador (BA), Brasil. Orcid: https://orcid. org/0000-0003-39065170

aquino@ufba.br

3 Universidade Federal da Bahia (UFBA), Instituto de Saúde Coletiva (ISC) -

Salvador (BA), Brasil.

Orcid: https://orcid.

org/0000-0001-7283-

2947

medina@ufba.br
RESUMO A Assistência Farmacêutica integra os serviços de Atenção Primária à Saúde, contribuindo para o controle de doenças com grande impacto à saúde, como a tuberculose. Para tanto, os serviços farmacêuticos devem contar com estrutura adequada nas unidades de atenção básica e com equipes que desenvolvam processos de trabalho qualificados, sendo esses atributos importantes objetos de estudos de avaliação. Este trabalho é o resultado de uma avaliação orientada por critérios e padrões de qualidade de estrutura e processo de trabalho de serviços farmacêuticos no cuidado ao paciente com tuberculose em duas unidades de saúde (A e B) de Salvador, Bahia, por meio de observação sistemática e entrevistas semiestruturadas direcionadas a farmacêuticos, gerentes e amostra de pacientes. $\mathrm{O}$ estudo demonstrou que a unidade A apresentou deficiências na estrutura, ao contrário da unidade B. No processo de trabalho, os serviços farmacêuticos eram pouco desenvolvidos na unidade A e plenamente desenvolvidos na unidade B. Pode-se observar a relação entre estrutura e processo de trabalho dos serviços farmacêuticos no cuidado ao paciente com tuberculose, pois a farmácia com maior deficiência na estrutura apresentou baixa consolidação no processo de trabalho dos farmacêuticos; e a farmácia com melhor estrutura apresentou consolidação plena.

PALAVRAS-CHAVE Assistência Farmacêutica. Estrutura dos serviços. Avaliação de processo. Tuberculose.

ABSTRACT The Pharmaceutical Assistance integrates Primary Health Care services, contributing to the control of diseases, such as tuberculosis, with great impact on health. In order for that to happen, the pharmaceutical services must have adequate structure in its basic care units, as well as teams to develop and to process qualified work, which are important attributes for the evaluation studies. This work is the result of an evaluation guided by structured quality criteria and standards, and pharmaceutical work process in order to assist patients with tuberculosis in two health units (A and B) in Salvador-Bahia, through systematic observation, semi-structured interviews directed to pharmacists and managers, and patients' samples. The result of this study showed that unit A presented deficiencies in structure, unlike unit $B$. In the work process, the pharmaceutical services were poorly developed in unit $A$, but fully developed in unit B. It was possible to observe a clear 
relation between the structure and the work process in the pharmacy services for the care of the patient with tuberculosis: the pharmacy with the greatest deficiency in the structure presented low consolidation in the work process, and the pharmacy with the best structure presented full consolidation in the pharmaceutical services.

KEYWORDS Pharmaceutical services. Structure of services. Process Assessment (Health Care). Tuberculosis.

\section{Introdução}

As diretrizes do Programa Nacional de Controle da Tuberculose (PNCT) preconizam que a Atenção Primária à Saúde (APS), estando mais próxima dos pacientes, de suas famílias e da comunidade onde vivem, deve ser o locus preferencial para o desenvolvimento de ações que tenham impacto na redução da doença, especialmente, no aumento da adesão dos pacientes ao tratamento. Entretanto, muitas unidades de APS não realizam essas ações que, em muitos municípios, são desenvolvidas em centros e hospitais de referência, fazendo com que a descentralização das ações de vigilância e controle de tuberculose permaneçam como um grande desafio no Brasil.

O cuidado ao paciente com tuberculose na APS engloba ações de prevenção, diagnóstico e tratamento, incluindo a oferta oportuna dos medicamentos necessários ${ }^{1}$. A disponibilidade de medicamentos deve ser assegurada pela Assistência Farmacêutica (AF), que deve estar integrada aos demais serviços de saúde, para a promoção do acesso e do uso racional de medicamentos, por meio da assistência ao paciente com enfoque na efetividade e na segurança da terapêutica, e da difusão das informações sobre medicamentos na perspectiva da educação em saúde e educação permanente das equipes ${ }^{2}$.

No âmbito da APS, a AF deve englobar um conjunto de práticas que envolvem atividades de regulação, planejamento, distribuição e dispensação de medicamentos essenciais, sendo assim, é um serviço que requer planejamento e inserção nos espaços da gestão para que seja assegurada a sua implementação e sustentabilidade 3 . Nesse cenário, o profissional farmacêutico deve estar inserido na equipe multidisciplinar de saúde, comprometendo-se não só com as atividades relacionadas com o processo de programação e aquisição como também com a utilização adequada desses medicamentos ${ }^{4}$.

Alguns estudos têm identificado problemas na implementação das ações da AF, quais sejam: as práticas com enfoque muito maior no gerenciamento do medicamento do que na assistência direta ao usuário ${ }^{5}$; as dificuldades que grande parte dos municípios brasileiros apresentam para implementar ações de $\mathrm{AF}$ no âmbito da APS, em virtude das condições inapropriadas para organização dos medicamentos nas farmácias ${ }^{4}$; as atividades são desenvolvidas nos serviços de APS, de forma geral, em espaços reduzidos, destinados apenas ao armazenamento de medicamentos ou espaços compartilhados para realização de diversas atividades, indicando condições inadequadas para o desenvolvimento de serviços de dispensação dos medicamentos. Além disso, há outros problemas estruturais, como presença de infiltrações, bolores nas paredes, fissuras no piso, bem como falta de claridade e ventilação ${ }^{6,7}$. 
Especialmente, no que diz respeito à garantia do acesso aos medicamentos essenciais, entre os quais se incluem os de tuberculose, há municípios que apresentam baixa disponibilidade e descontinuidade da oferta, além de problemas na sua dispensação em virtude da baixa qualificação dos profissionais envolvidos para a devida orientação aos pacientes 4 .

Analisando-se os estudos disponíveis sobre avaliação de serviços farmacêuticos na APS abordando aspectos relacionados com a estrutura e com o processo de trabalho do profissional farmacêutico, verificou-se que poucos discorrem sobre o tema, e nenhum deles aborda especificamente o cuidado farmacêutico ao paciente com tuberculose. Considerando-se a relevância desse tema e a magnitude da doença para a capital baiana, o objetivo deste estudo foi avaliar a estrutura dos serviços farmacêuticos em Unidades Básicas de Saúde (UBS) e o processo de trabalho dos profissionais farmacêuticos no cuidado ao paciente com tuberculose na APS no município de Salvador, Bahia.

\section{Material e métodos}

Trata-se de um estudo avaliativo, de desenho qualitativo, sobre a qualidade da AF no cuidado ao paciente com tuberculose, desenvolvido em duas unidades de atenção primária em Salvador, Bahia.

Foi elaborado um modelo de avaliação que compreende as duas dimensões da AF: técnico-gerencial e técnico-assistencial. A dimensão técnico-gerencial envolve as atividades administrativas relacionadas com a seleção, programação, aquisição, armazenamento e distribuição de medicamentos, enquanto a dimensão técnico-assistencial engloba as atividades de dispensação, orientação farmacêutica e seguimento farmacoterapêutico ${ }^{8}$. Essas duas dimensões apresentam uma forte relação, visto que as atividades gerenciais são essenciais para o funcionamento da farmácia, e as assistenciais são desenvolvidas com enfoque no cuidado e orientação ao usuário?.

Para cada uma das dimensões, foi definido um conjunto de critérios e padrões relacionados com os atributos de estrutura dos serviços farmacêuticos e com os processos desenvolvidos pelos farmacêuticos no cuidado ao paciente, a partir dos indicadores do método para aplicação do planejamento da AF - Planejar é preciso $^{10}$ e dos estudos de Barreto e Guimarães ${ }^{3}$ e Correia, Mota, Arrais et al.11.

Os critérios referentes à estrutura corresponderam às instalações prediais, recursos materiais e recursos humanos necessários para o desenvolvimento das atividades inseridas nas duas dimensões. Os critérios referentes ao processo de trabalho foram definidos da seguinte forma: na dimensão técnico-gerencial, foram estabelecidos os critérios de programação para solicitação de medicamentos, forma de organização dos medicamentos e registro e conferência de controle de estoque (registro de informações); já na dimensão técnico-assistencial, foram definidos os critérios de entrega do medicamento ao paciente conforme prescrição, fornecimento de informações aos pacientes sobre o uso de medicamentos, fornecimento de informações sobre possíveis Reações Adversas a Medicamentos (RAM) e interações, realização de anamnese, registro e preenchimento do prontuário, intervenção farmacêutica, interação/articulação do profissional farmacêutico com os demais profissionais do serviço de cuidado ao paciente com tuberculose e relação do farmacêutico com os níveis da gestão. Destaca-se que foram estabelecidas regras de pontuações, em percentual, para os critérios e padrões definidos, no sentido de nortear a análise dos dados, as quais estão descritas no quadro 1 . 
Quadro 1. Critérios e padrões de estrutura e processo de trabalho, considerando-se as duas dimensões definidas no estudo, a dos serviços farmacêuticos técnico-gerenciais e a dos técnico-assistenciais

Dimensões Criterios

Padrões

1 Sala contendo espaço suficiente para armazenamento dos medicamentos;

zacionais: instalações prediais

Ambiente climatizado com ar-condicionado (temperatura entre $15^{\circ} \mathrm{C}$ e $30^{\circ} \mathrm{C}$, e umidade relativa em até $70 \%$ ).

Recursos materiais: equipamentos e mobiliários

Pallets, estantes e/ou armários;

Geladeira exclusiva para acondicionamento dos medicamentos termolábeis;

1 computador com internet por estação de atendimento;

Escaninhos e prateleiras para acondicionar os medicamentos;

1 computador com internet na sala do farmacêutico:

1 estação de atendimento (guichê) para cada auxiliar;

1 cadeira por estação de atendimento para o atendente;

1 mesa para cada sala; cia, mas que ocasionaria problemas ergonômicos (80\%); ou Não existência ( $0 \%)$

1 cadeira para o farmacêutico.

\begin{tabular}{ll}
\hline $\begin{array}{l}\text { Recursos ma- } \\
\text { teriais: material }\end{array}$ & 1Relação Nacional de Medicamentos Essenciais; \\
técnico e sof- & 1 Manual de Boas Práticas de Armazenamento; \\
tware disponível & 1 Plano de Gerenciamento de Resíduos de Serviços de \\
para suporte ao & Saúde (PGRSS); \\
serviço & $\begin{array}{l}\text { Sistema/software utilizado para gerenciamento dos } \\
\text { estoques dos medicamentos. }\end{array}$
\end{tabular}

Recursos Huma- 1 profissional farmacêutico, para atendimento aos pa-

nos

cientes com tuberculose, em todo o horário de funciona-

Existência (100\%) ou Não

mento da unidade;

1 auxiliar administrativo por guichê.

\begin{tabular}{lll}
\hline Programação para & Utilização do método Consumo Histórico para realizar a & Utilização (100\%) ou Não \\
solicitação de & programação; & utilização (0\%). \\
medicamentos & Utilização de métodos Perfil Epidemiológico ou Oferta & \\
& Ajustada para realizar a programação.
\end{tabular}

Forma de organi- 1. Conferência dos medicamentos no recebimento da Realização total (100\%);

zação dos medi- programação mensal;

camentos

2. Organização dos medicamentos em pallets, estantes

Realização parcial (50\%) ou

Não realização $(0 \%)$ e/ou armários:

3. Acondicionamento adequado dos medicamentos termolábeis em geladeira;

4. Conservação dos medicamentos em condições adequadas para assegurar as suas características físico-químicas;

5. Medicamentos identificados com: nome da substân- Pontuação de 20\% para cia, dosagem, apresentação, lote e data de validade. cada item analisado

Registro e confe- $\quad$ 1. Conferência, registro e controle da movimentação Pontuação de $25 \%$ para cada

rência de controle física, registro de entrada e registro de saída dos medica- atividade analisada.

de estoque (re- mentos, de forma sistemática;

gistro de informa- 2. Utilização de sistema informatizado. ções)
Utilização (100\%) ou Não

utilizacão (0\%). 


\section{Quadro 1. (cont.)}

\begin{tabular}{|c|c|c|c|}
\hline \multirow{2}{*}{ 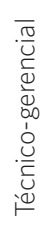 } & \multirow[t]{2}{*}{$\begin{array}{l}\text { Relação do farma- } \\
\text { cêutico com os } \\
\text { níveis da gestão }\end{array}$} & $\begin{array}{l}\text { Existência de comunicação do profissional farmacêutico } \\
\text { com o nível da gerência da UBS para resolução de pro- } \\
\text { blemas técnico-gerenciais; }\end{array}$ & \multirow[t]{2}{*}{$\begin{array}{l}\text { Existência (100\%) ou Não } \\
\text { existência (0\%). }\end{array}$} \\
\hline & & $\begin{array}{l}\text { Existência de comunicação do profissional farmacêutico } \\
\text { com o nível central da SMS para resolução de problemas } \\
\text { técnico-gerenciais. }\end{array}$ & \\
\hline \multirow{21}{*}{ 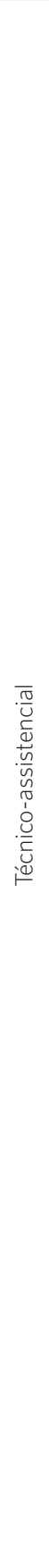 } & \multirow{3}{*}{$\begin{array}{l}\text { Recursos organi- } \\
\text { zacionais: instala- } \\
\text { ções prediais }\end{array}$} & $\begin{array}{l}\text { 1Sala contendo espaço suficiente para dispensação dos } \\
\text { medicamentos; }\end{array}$ & \multirow{3}{*}{$\begin{array}{l}\text { Existência (100\%); Existên- } \\
\text { cia insuficiente ( } 50 \%) \text {; ou } \\
\text { Não existência (0\%). }\end{array}$} \\
\hline & & $\begin{array}{l}01 \text { Sala contendo espaço suficiente para realizar orienta- } \\
\text { ção farmacêutica aos pacientes; }\end{array}$ & \\
\hline & & $\begin{array}{l}\text { Ambiente climatizado com ar-condicionado (temperatu- } \\
\text { ra entre } 15^{\circ} \mathrm{C} \text { a } 30^{\circ} \mathrm{C} \text {, e umidade relativa em até } 70 \% \text { ). }\end{array}$ & \\
\hline & \multirow{6}{*}{$\begin{array}{l}\text { Recursos mate- } \\
\text { riais: equipamen- } \\
\text { tos e mobiliários }\end{array}$} & Pallets, estantes e/ou armários; & \multirow{4}{*}{$\begin{array}{l}\text { Existência (100\%) ou Não } \\
\text { existência (0\%). }\end{array}$} \\
\hline & & 1 computador com internet por estação de atendimento; & \\
\hline & & $\begin{array}{l}\text { Escaninhos e prateleiras para acondicionar os medica- } \\
\text { mentos; }\end{array}$ & \\
\hline & & $\begin{array}{l}1 \text { computador com internet na sala de orientação farma- } \\
\text { cêutica; }\end{array}$ & \\
\hline & & 1 cadeira para o farmacêutico; & Existência (100\%); Existên- \\
\hline & & $\begin{array}{l}1 \text { cadeira para o paciente na sala de orientação farma- } \\
\text { cêutica. }\end{array}$ & $\begin{array}{l}\text { cia, mas que ocasionaria pro- } \\
\text { blemas ergonômicos ( } 80 \%) \text {; } \\
\text { ou Não existência ( } 0 \% \text { ). }\end{array}$ \\
\hline & \multirow{4}{*}{$\begin{array}{l}\text { Recursos ma- } \\
\text { teriais: material } \\
\text { técnico e sof- } \\
\text { tware disponível } \\
\text { para suporte ao } \\
\text { serviço }\end{array}$} & 1 Formulário Nacional Terapêutico; & \multirow{4}{*}{$\begin{array}{l}\text { Existência (100\%) ou Não } \\
\text { existência (0\%). }\end{array}$} \\
\hline & & 1 Relação Nacional de Medicamentos Essenciais; & \\
\hline & & $\begin{array}{l}1 \text { Manual de Recomendações para o controle da Tuber- } \\
\text { culose no Brasil; }\end{array}$ & \\
\hline & & $\begin{array}{l}\text { Sistema/software utilizado para registro de dispensação } \\
\text { dos medicamentos aos pacientes. }\end{array}$ & \\
\hline & \multirow{2}{*}{$\begin{array}{l}\text { Recursos ma- } \\
\text { teriais: material } \\
\text { educativo para os } \\
\text { pacientes }\end{array}$} & $\begin{array}{l}\text { Cartilhas, folhetos, folders elaborados pela Assistência } \\
\text { Farmacêutica para orientação aos pacientes na adesão } \\
\text { ao tratamento da tuberculose; }\end{array}$ & \multirow[t]{2}{*}{$\begin{array}{l}\text { Existência (100\%) ou Não } \\
\text { existência (0\%) }\end{array}$} \\
\hline & & $\begin{array}{l}\text { Cartilhas, folhetos, folders elaborados pelo Programa de } \\
\text { Controle da Tuberculose para orientação aos pacientes } \\
\text { na adesão ao tratamento da tuberculose. }\end{array}$ & \\
\hline & \multirow[t]{2}{*}{$\begin{array}{l}\text { Recursos Huma- } \\
\text { nos }\end{array}$} & $\begin{array}{l}1 \text { profissional farmacêutico, para atendimento aos pa- } \\
\text { cientes com tuberculose, em todo o horário de funciona- } \\
\text { mento da unidade; }\end{array}$ & \multirow[t]{2}{*}{$\begin{array}{l}\text { Existência (100\%) ou Não } \\
\text { existência (0\%). }\end{array}$} \\
\hline & & 1 auxiliar administrativo por guichê. & \\
\hline & $\begin{array}{l}\text { Entrega do medi- } \\
\text { camento ao pa- } \\
\text { ciente conforme } \\
\text { prescrição }\end{array}$ & $\begin{array}{l}\text { Leitura da prescrição para o paciente em todas as dis- } \\
\text { pensações. }\end{array}$ & $\begin{array}{l}\text { Realização total (100\%); } \\
\text { Realização parcial (50\%) ou } \\
\text { Não realização (0\%). }\end{array}$ \\
\hline & \multirow{3}{*}{$\begin{array}{l}\text { Fornecimento de } \\
\text { informações aos } \\
\text { pacientes sobre } \\
\text { o uso de medica- } \\
\text { mentos }\end{array}$} & $\begin{array}{l}\text { Orientação sobre a utilização dos medicamentos, como } \\
\text { modo de uso em todas as dispensações; }\end{array}$ & \multirow{3}{*}{$\begin{array}{l}\text { Realização total (100\%); } \\
\text { Realização parcial (50\%) ou } \\
\text { Não realização (0\%). }\end{array}$} \\
\hline & & $\begin{array}{l}\text { Orientação sobre a utilização dos medicamentos, como } \\
\text { via de administração em todas as dispensações; }\end{array}$ & \\
\hline & & $\begin{array}{l}\text { Orientação sobre a utilização dos medicamentos, como } \\
\text { posologia e horário de uso em todas as dispensações. }\end{array}$ & \\
\hline
\end{tabular}


Quadro 1. (cont.)

\begin{tabular}{|c|c|c|c|}
\hline \multirow{7}{*}{ 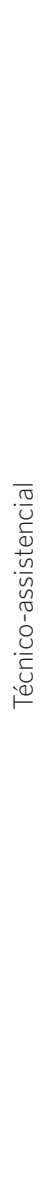 } & $\begin{array}{l}\text { Fornecimento } \\
\text { de informações } \\
\text { sobre possíveis } \\
\text { Reações Adversas } \\
\text { a Medicamentos } \\
\text { (RAM) e intera- } \\
\text { ções }\end{array}$ & $\begin{array}{l}\text { Orientação para todos os pacientes, sobre as possíveis } \\
\text { reações adversas na primeira dispensação de cada es- } \\
\text { quema terapêutico. } \\
\text { Orientação para todos os pacientes, sobre as interações } \\
\text { medicamentosas, na primeira dispensação de cada } \\
\text { esquema terapêutico. }\end{array}$ & $\begin{array}{l}\text { Realização total (100\%); } \\
\text { Realização parcial (50\%) ou } \\
\text { Não realização (0\%). }\end{array}$ \\
\hline & $\begin{array}{l}\text { Realização de } \\
\text { anamnese }\end{array}$ & $\begin{array}{l}\text { Aplicação de entrevista aos pacientes para obter infor- } \\
\text { mações sobre as características intrínsecas, estilo de } \\
\text { vida, patologias e tratamentos coexistentes na admissão } \\
\text { do paciente. }\end{array}$ & $\begin{array}{l}\text { Realização total (100\%); } \\
\text { Realização parcial (50\%) ou } \\
\text { Não realização (0\%). }\end{array}$ \\
\hline & $\begin{array}{l}\text { Registro e pre- } \\
\text { enchimento do } \\
\text { prontuário }\end{array}$ & $\begin{array}{l}\text { Prontuário preenchido com as informações obtidas e } \\
\text { atualizadas em todas as dispensações. }\end{array}$ & $\begin{array}{l}\text { Realização total (100\%); } \\
\text { Realização parcial (50\%) ou } \\
\text { Não realização (0\%). }\end{array}$ \\
\hline & $\begin{array}{l}\text { Intervenção far- } \\
\text { macêutica }\end{array}$ & $\begin{array}{l}\text { Identificação de problemas relacionados a medicamen- } \\
\text { tos e execução de plano de intervenção farmacêutica, } \\
\text { quando necessário. }\end{array}$ & $\begin{array}{l}\text { Realização total (100\%); } \\
\text { Realização parcial (50\%) ou } \\
\text { Não realização (0\%). }\end{array}$ \\
\hline & $\begin{array}{l}\text { Interação/articu- } \\
\text { lação do profis- } \\
\text { sional farmacêuti- } \\
\text { co com os demais } \\
\text { profissionais do } \\
\text { serviço de cuida- } \\
\text { do ao paciente } \\
\text { com tuberculose }\end{array}$ & $\begin{array}{l}\text { Existência de mecanismos junto aos demais profissionais } \\
\text { envolvidos no controle da tuberculose para resolução de } \\
\text { problemas dos pacientes. }\end{array}$ & $\begin{array}{l}\text { Existência (100\%) ou Não } \\
\text { existência (0\%). }\end{array}$ \\
\hline & \multirow[t]{2}{*}{$\begin{array}{l}\text { Relação do farma- } \\
\text { cêutico com os } \\
\text { níveis da gestão }\end{array}$} & $\begin{array}{l}\text { Existência de comunicação do profissional farmacêutico } \\
\text { com o nível da gerência da UBS para resolução de pro- } \\
\text { blemas técnico-assistenciais. }\end{array}$ & \multirow[t]{2}{*}{$\begin{array}{l}\text { Existência (100\%) ou Não } \\
\text { existência (0\%). }\end{array}$} \\
\hline & & $\begin{array}{l}\text { Existência de comunicação do profissional farmacêutico } \\
\text { com o nível central da SMS para resolução de problemas } \\
\text { técnico-assistenciais. }\end{array}$ & \\
\hline
\end{tabular}

Fonte: Elaboração própria, com base em Barreto e Guimarães ${ }^{3}$, Brasil|8 e Correia, Mota, Arrais, et al."1

A partir do modelo, foi elaborado um instrumento que foi validado por um grupo composto por 18 especialistas, farmacêuticos com experiência de atuação na gestão e no cuidado da AF, por meio da técnica de consenso pelo Método Delfos. Nesse processo, foi distribuída para cada participante uma proposta inicial de critérios e padrões. Os participantes foram convidados a analisar o instrumento e atribuir pesos aos critérios e padrões propostos ou sugerir alterações nas suas descrições. As respostas obtidas foram tratadas estatisticamente e repassadas para o conhecimento de todos. Com o aprimoramento do instrumento, foi realizada uma segunda rodada, na qual os participantes atribuíram os pesos finais aos critérios e padrões, realizando-se então um segundo tratamento estatístico. De acordo com Souza, Vieira da Silva e Hartz ${ }^{\mathbf{1 2}}$, novas rodadas e análises podem ser realizadas até que seja obtido um grau de consenso considerado satisfatório. No presente estudo, duas rodadas foram suficientes.

O estudo foi realizado em duas UBS, e a unidade de análise correspondeu aos serviços de AF dessas UBS, as quais desenvolviam ações de controle de tuberculose, bem como disponibilizavam medicamentos aos pacientes, contando-se com a presença do profissional farmacêutico.

As unidades de saúde foram selecionadas considerando-se a magnitude do número de casos notificados de tuberculose, bem 
como a consolidação das ações de AF para o cuidado ao paciente com tuberculose. As informações relacionadas com a magnitude do número de casos notificados da doença foram obtidas por meio do Tabnet-Salvador ${ }^{\mathbf{1 3}}$; e, após consulta e colaboração do nível central da AF, foram selecionadas duas unidades de saúde, unidades $\mathrm{A}$ e $\mathrm{B}$, as quais apresentavam realidades distintas em relação à consolidação dos serviços farmacêuticos. A unidade A encontrava-se em fase inicial de consolidação dos serviços farmacêuticos no cuidado ao paciente com tuberculose; e a unidade B já tinha esses serviços consolidados.

A coleta dos dados foi realizada pelo pesquisador, no período de dezembro de 2013 a abril de 2014, por meio de entrevistas semiestruturadas feitas com os farmacêuticos do nível central na Secretaria de Saúde, com os farmacêuticos e com os gerentes das unidades selecionadas, bem como com uma amostra de pacientes, definida a partir do atendimento médio diário nessas unidades. $\mathrm{Na}$ Coordenação Municipal de AF, foram entrevistados os profissionais farmacêuticos responsáveis pela gestão da AF. Nas unidades de saúde A e $\mathrm{B}$, foram entrevistados todos os farmacêuticos atuantes, sendo dois profissionais em cada unidade, seus respectivos gerentes e dois grupos de pacientes em atendimento, sendo seis pacientes em cada unidade, correspondendo a uma amostra por conveniência. Para a composição dessa amostra, foi definido como critério os pacientes estarem em uso do esquema básico (fase I ou II) do tratamento de tuberculose, excluindo-se aqueles que estivessem realizando quimioprofilaxia. Foi realizada, também, observação sistemática nas duas farmácias, guiada por um roteiro.

Os dados obtidos foram tabulados em planilhas no software Excel $^{\circledR}$, procedendo-se à análise dos critérios e padrões estabelecidos no estudo, os quais tiveram pontuações atribuídas, em percentual, posteriormente sumarizados em gráficos em forma de radar.

O estudo foi aprovado pelo Comitê de Ética do Instituto de Saúde Coletiva da Universidade Federal da Bahia, conforme parecer consubstanciado do CEP no 490.975 , e teve a anuência da Secretaria Municipal de Saúde de Salvador, para a realização da coleta dos dados. As entrevistas foram realizadas após esclarecimento de cada informante quanto aos propósitos do estudo, leitura e assinatura do Termo de Consentimento Livre e Esclarecido.

\section{Resultados}

A rede de APS do município de Salvador, no momento em que o estudo foi realizado, era composta por 115 unidades básicas, incluindo Unidades de Saúde da Família (USF). No que diz respeito ao cuidado ao paciente com tuberculose, de acordo com informações obtidas por meio da Coordenação de Assistência Farmacêutica da Secretaria Municipal de Saúde, 70\% das unidades realizavam dispensação dos medicamentos para o tratamento da tuberculose; e, dessas, apenas cerca de $30 \%$ contavam com o profissional farmacêutico para desenvolver as atividades de cuidado aos pacientes na dispensação dos medicamentos (tabela 1). 
Tabela 1. Número total e por tipo de unidades de saúde de APS, e número e percentual de unidades com realização de dispensação de medicamentos para tuberculose (TB) e disponibilidade de farmacêutico por Distrito Sanitário. SalvadorBA, 2013

\begin{tabular}{|c|c|c|c|c|c|}
\hline \multirow{2}{*}{ Distrito Sanitário } & \multicolumn{3}{|c|}{ № Unidades de Saúde APS } & \multirow{2}{*}{$\begin{array}{l}\text { № e \% Unidades } \\
\text { dispensadoras de } \\
\text { medicamentos TB }\end{array}$} & \multirow{2}{*}{$\begin{array}{r}\text { № e \% Unidades } \\
\text { dispensadoras com } \\
\text { farmacêutico }\end{array}$} \\
\hline & CS/ Posto/ UBS & PSF & TOTAL & & \\
\hline Barra/Rio Vermelho & 5 & 4 & 9 & $7(77,7)$ & $3(42,8)$ \\
\hline Boca do Rio & 3 & 2 & 5 & $4(80,0)$ & $1(25,0)$ \\
\hline Brotas & 4 & 1 & 5 & $3(60,0)$ & $1(33,3)$ \\
\hline Cabula/Beiru & 14 & 3 & 17 & $9(52,9)$ & $3(33,3)$ \\
\hline Cajazeiras & 1 & 7 & 8 & $7(87,5)$ & $0(0,0)$ \\
\hline Centro Histórico & 7 & 2 & 9 & $9(100,0)$ & $4(44,4)$ \\
\hline Itapagipe & 3 & 2 & 5 & $3(60,0)$ & $1(33,3)$ \\
\hline Itapuã & 4 & 3 & 7 & $5(71,4)$ & $3(60,0)$ \\
\hline Liberdade & 3 & 2 & 5 & $4(80,0)$ & $4(100,0)$ \\
\hline Pau da Lima & 9 & 1 & 10 & $3(30,0)$ & $2(66,6)$ \\
\hline São Caetano/Valéria & 4 & 7 & 11 & $8(72,7)$ & $1(12,5)$ \\
\hline Subúrbio Ferroviário & 5 & 19 & 24 & $19(79,2)$ & $1(5,3)$ \\
\hline TOTAL & 62 & 53 & 115 & $81(70,0)$ & $24(29,6)$ \\
\hline
\end{tabular}

Fonte: Elaboração própria, com base em Salvador-SMS13.

As unidades de saúde onde o estudo foi realizado eram consideradas serviços de referência para atenção ao paciente com tuberculose nos respectivos distritos sanitários, no entanto, os programas de controle da doença nas duas unidades funcionavam de formas distintas. Na unidade A, verificou-se que, na fase inicial do tratamento, o paciente recebia o medicamento na sala do programa de controle da tuberculose, coordenado por enfermeiros, onde recebia orientações quanto ao tratamento e à dose supervisionada. Nos meses seguintes, o paciente se dirigia à farmácia para recebimento dos medicamentos, após a consulta com os profissionais de enfermagem. Na unidade B, os pacientes tinham suas consultas agendadas com os médicos e com os enfermeiros e, posteriormente, eram atendidos pelos farmacêuticos para recebimento dos medicamentos e das devidas orientações.

Em relação às farmácias, destaca-se que a unidade A dispunha de um ambiente único para desenvolvimento de todas as atividades farmacêuticas, além da área para acesso dos pacientes aos guichês de dispensação. Diferentemente, a farmácia da unidade B era dividida em quatro ambientes: a sala de armazenamento de medicamentos, a área de dispensação de medicamentos, a sala de orientação farmacêutica e uma copa, além da área para acesso dos pacientes (figura 1). 
Farmácia da unidade $\mathrm{A}$

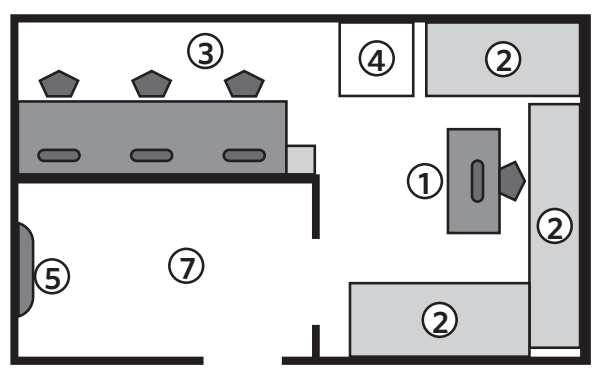

1 - Sala de armazenamento dos medicamentos;

2 - Prateleiras para armazenamento:

3 - Sala para dispensação dos medicamentos;

4 - Geladeira;

5 - Ar-condicionado;

6 - Estantes com escaninhos;

7 - Área de acesso dos pacientes:

8 - Sala de orientação farmacêutica;

9 - Copa.

Fonte: Elaboração própria.
Farmácia da unidade B

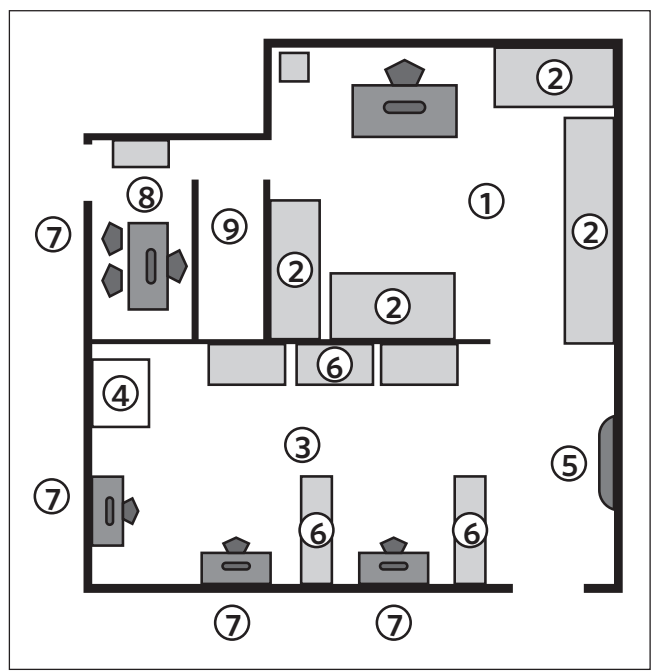

No que diz respeito à dimensão técnico-gerencial, quanto ao processo de trabalho, observaram-se semelhanças entre as duas unidades, embora a unidade B tenha apresentado melhor desempenho. Em relação à estrutura, verificou-se que a unidade A apresentou deficiências nas instalações prediais, nos equipamentos e nos mobiliários, embora tenha sido reformada poucos meses antes da realização deste estudo (figura 2). Nessa unidade, observou-se a inexistência de espaços delimitados para o desenvolvimento das atividades de conferência, de armazenamento e de movimentação de estoque dos medicamentos. Um dos farmacêuticos da unidade A destacou que

há dificuldade em relação ao layout da farmácia, que apesar de ter sofrido reforma há pouco tempo, não ajudou porque não foi consultada a parte técnica, como deveria, para prestar o serviço.

Corroborando essa fala, o outro profissional farmacêutico da unidade salientou consequências relacionadas com a inexistência de espaços, conforme descrito a seguir:

Estamos sem área de recebimento de medicamentos, sem almoxarifado, então todos os medicamentos que a gente recebe ficam aqui. Como temos que fazer a conferência antes de colocar junto com os medicamentos que já estavam, normalmente eu faço o possível para esvaziar as prateleiras, mas isso não resolve o problema, e coloco sempre no topo das prateleiras.

$\mathrm{Na}$ farmácia da unidade $\mathrm{B}$, havia poucas deficiências quanto às instalações prediais, para o desenvolvimento dos serviços farmacêuticos técnico-gerenciais, visto que contava com os espaços adequados a essas atividades, ainda que com necessidades de reformas e de substituição de equipamentos e mobiliários (figura 2).

Em relação aos materiais técnicos e software para suporte ao serviço, as duas farmácias apresentaram realidades 
semelhantes: ambas dispunham de grande parte dos materiais necessários para o desenvolvimento dos serviços. No que diz respeito aos recursos humanos, verificaram-se semelhanças entre as duas farmácias, tanto em relação à existência do profissional farmacêutico durante todo o horário de funcionamento como dos atendentes nos guichês em quantidades suficientes (figura 2).

Figura 2. Gráficos comparativos acerca da avaliação de estrutura e processo de trabalho na dimensão técnico-gerencial para o cuidado ao paciente com tuberculose nas farmácias das Unidades A e B

\section{Unidade A}

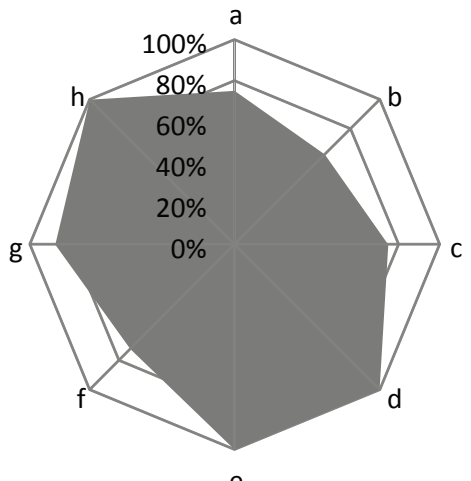

\section{Unidade B}

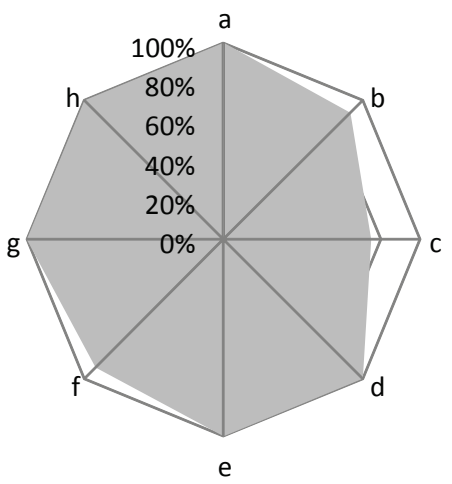

a) Recursos organizacionais: instalações prediais

b) Recursos materiais: equipamentos e mobiliários

c) Recursos materiais: material técnico e software

d) Recursos humanos

e) Programação para solicitação de medicamentos

f) Forma de organização dos medicamentos

g) Registro e conferência de controle de estoque

h) Relação do farmacêutico com os níveis da gestão
Em se tratando da dimensão técnico-assistencial, verificou-se, em relação à estrutura, que a farmácia da unidade A não contava com um espaço delimitado para realizar a dispensação e a orientação farmacêutica ao paciente com tuberculose, ao contrário da unidade $\mathrm{B}$, que contava com uma sala específica para atendimento a esses pacientes. Destaca-se ainda que, diferentemente da unidade $\mathrm{B}$, a unidade A não dispunha de todos os materiais educativos para os pacientes, apresentando, portanto, desempenho parcial em relação a esse critério (figura 3). 
Figura 3. Gráficos comparativos acerca da avaliação de estrutura e processo de trabalho na dimensão técnico-assistencial para o cuidado ao paciente com tuberculose nas farmácias das Unidades A e B

\section{Unidade A}

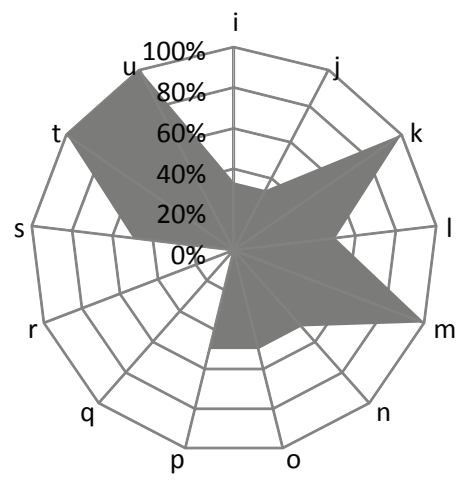

Fonte: Elaboração própria.

\section{Unidade B}

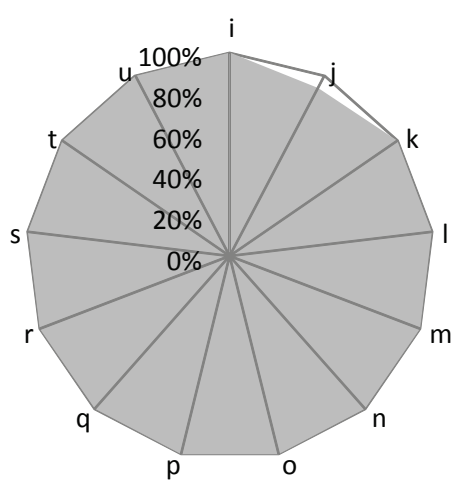

i) Recursos organizacionais: instalações prediais

j) Recursos materiais: equipamentos e mobiliários

k) Recursos materiais: material técnico e software

l) Recursos materiais: material educativo para os pacientes

m) Recursos humanos

n) Entrega do medicamento ao paciente conforme prescrição

o) Fornecimento de informações sobre o uso de medicamentos

p) Fornecimento de informações sobre RAM e interações

q) Realização de anamnese

r) Registro e preenchimento do prontuário

s) Intervenção farmacêutica

t) Interação do profissional farmacêutico com os demais profissionais

u) Relação do farmacêutico com os níveis da gestão
Quanto ao processo de trabalho na dimensão técnico-assistencial, observou-se que a entrega, o fornecimento de informações sobre o uso e possíveis reações adversas de medicamentos, a realização de anamnese e registro de preenchimento do prontuário e a intervenção farmacêutica eram desenvolvidos plenamente na unidade $B$, sendo pouco executados na unidade A. No presente estudo, verificou-se que os farmacêuticos da unidade B apresentavam autonomia para realizar intervenções farmacêuticas, quando necessário, ao contrário dos profissionais da unidade A (figura 3).

Em relação à interação/articulação dos farmacêuticos com os demais profissionais do serviço de cuidado ao paciente com tuberculose, verificou-se que, na unidade A, essa ação era pouco consolidada, diferentemente da unidade B, plenamente consolidada, pois os farmacêuticos participavam do planejamento de todas as atividades propostas para o desenvolvimento do programa. Quanto à relação desses profissionais com os níveis da gestão, observou-se que, nas duas unidades, os farmacêuticos mantinham boa comunicação com a gerência e com a coordenação do nível central da AF (figura 3).

\section{Discussão}

A rede de atenção básica do Município de Salvador, em relação ao cuidado ao paciente com tuberculose, está em desacordo com as diretrizes do $\mathrm{PNCT}^{1}$, que preconizam que o cuidado ao paciente com tuberculose deve ser assegurado em $100 \%$ das unidades de APS, tendo em vista a ênfase na descentralização das medidas de controle da doença para esse nível de atenção à saúde. No município, cerca de um terço das unidades básicas não realizava uma das principais ações de controle da doença, que é a dispensação dos medicamentos. Além disso, entre as unidades que dispensavam medicamentos, a maioria não contava com profissionais farmacêuticos, os quais deveriam ser responsáveis por essa atividade, sendo bastante desigual a distribuição das unidades básicas que dispunham desses profissionais entre os distritos sanitários.

Diante disso, faz-se necessário estabelecer a relação dos serviços farmacêuticos com a APS, reconhecendo que suas ações devem ser desenvolvidas pelo profissional farmacêutico incorporado à equipe de saúde com vistas 
à melhoria da qualidade de vida da população ${ }^{2}$. Destaca-se que o farmacêutico é capaz de conhecer as atividades que compõem a AF, de deter e de disponibilizar informações técnicas sobre os medicamentos, de aconselhar pacientes, além de constituir a interface entre a prescrição e a dispensação dos medicamentos ${ }^{14}$. Para uma efetiva prática na APS, esse profissional deve conhecer o território em que atua, observando-se a população a que atende, bem como as suas características, no sentido de desenvolver todos os serviços farmacêuticos necessários para a otimização de uma farmacoterapia com corresponsabilidade ${ }^{15}$, estabelecendo diálogos com o paciente e com demais profissionais envolvidos no cuidado, especialmente com os pacientes com tuberculose.

Em relação às unidades de saúde avaliadas, observaram-se realidades distintas no desenvolvimento das atividades farmacêuticas, sendo constatadas deficiências na farmácia da unidade $\mathrm{A}$, que não atendeu plenamente aos critérios estabelecidos, ao contrário da farmácia da unidade B, que apresentou consolidação dos serviços farmacêuticos, cumprindo os critérios estabelecidos em sua quase totalidade.

$\mathrm{Na}$ avaliação dos critérios relacionados com a dimensão técnico-gerencial, as deficiências na estrutura na unidade A em maior proporção que na unidade $B$ podem ter sido fatores determinantes para uma menor execução de atividades como organização e controle de estoque dos medicamentos. A estocagem dos medicamentos tem como objetivo assegurar a qualidade destes, por meio de condições ambientais adequadas e do controle de estoque, de forma a garantir sua disponibilidade nas farmácias para os usuários ${ }^{16}$. A ausência de espaços adequados interfere no controle eficiente dos medicamentos, podendo acarretar problemas, como falta ou perdas por validade expirada.

Verificou-se que, em relação aos recursos materiais, tais como equipamentos e mobiliários, a unidade A apresentou mais deficiências em relação à unidade $\mathrm{B}$. De acordo com
Moura, Cunha, Fonseca et al..77, os equipamentos e mobiliários se inserem na estrutura necessária para propiciar a prestação dos serviços de saúde, os quais devem estar adequados para o conjunto de ações propostas, em que se incluem as ações de AF.

Quanto ao processo de trabalho na dimensão técnico-gerencial, o desempenho da unidade B foi melhor do que da unidade A no que diz respeito a todas as atividades: programação, aquisição, organização e controle de estoque dos medicamentos. Canabarro e Hahnn ${ }^{18}$ reforçam a necessidade de assegurar a qualidade dos medicamentos por meio das boas práticas de aquisição, armazenamento, conservação e dispensação, mediante incorporação do farmacêutico na equipe multidisciplinar da APS. Ressalta-se que, nas duas unidades avaliadas, contava-se com a presença de farmacêuticos, que realizavam os serviços farmacêuticos técnico-gerenciais. No entanto, a unidade A apresentava dificuldades para o seu desenvolvimento, provavelmente em decorrência da deficiência de estrutura e espaço.

Ao analisar os critérios relacionados com a dimensão técnico-assistencial, observou-se que a ausência de espaços reservados para a dispensação, para a orientação e para a intervenção farmacêutica na unidade A, provavelmente, contribuiu para o baixo desempenho das atividades. Realidade contrária foi evidenciada na farmácia da unidade B. De acordo com as Diretrizes para estruturação de farmácias no âmbito do SUS ${ }^{\mathbf{8}}$ o espaço físico para dispensação deve ter os equipamentos e mobiliários necessários para assegurar a logística, o fluxo de trabalho, bem como a capacidade de atendimento do serviço; ademais, o espaço destinado à orientação farmacêutica deve ser reservado, de forma que permita diálogo entre o paciente e o profissional, favorecendo o cuidado ao usuário.

A dispensação de medicamentos é compreendida como processo informativo referente ao tratamento, ao acompanhamento e à avaliação farmacoterapêutica da prescrição, requerendo, portanto, a presença do 
profissional farmacêutico, com o propósito de se aproximar das necessidades dos usuários, não se restringindo apenas à simples entrega de medicamentos, mas, sim, à promoção da sua utilização adequada, ${ }^{3,19}$. Alencar e Nascimento ${ }^{16}$ ressaltam a dispensação de medicamentos como a atividade que encerra a relação do usuário com a unidade de saúde após o atendimento clínico, constituindo-se em uma oportunidade de efetivar a adequada orientação em relação à prescrição, reduzindo, portanto, riscos ou falhas relacionadas com a terapêutica medicamentosa. $\mathrm{Na}$ farmácia da unidade B, foi possível observar o desenvolvimento dessas atividades, bem como os resultados positivos obtidos com os pacientes. As dificuldades observadas na farmácia da unidade $\mathrm{A}$ em relação à dispensação dos medicamentos podem ser reforçadas por Leite, Bernardo, Álvares et al. ${ }^{19}$ quando destacam que há forte limitação para o desenvolvimento dessa atividade como um serviço de saúde pelas deficiências de organização e estruturação dos serviços farmacêuticos, acentuadas pelo modelo focado na gestão do estoque de medicamentos.

A intervenção farmacêutica corresponde ao ato planejado, documentado e realizado com o usuário, com o objetivo de prevenir os erros de prescrição e de administração dos medicamentos. Dessa forma, o farmacêutico tem um papel fundamental na garantia da farmacoterapia adequada, buscando resultados terapêuticos seguros e minimizando os resultados desfavoráveis ${ }^{20-22}$. Nesse cenário, a troca de informações entre os profissionais da equipe de saúde mostra-se fundamental para a promoção do uso seguro dos medicamentos $^{23}$. No presente estudo, verificou-se que os farmacêuticos da unidade A tinham pouca autonomia para realizar intervenções farmacêuticas, quando necessário, ao contrário dos farmacêuticos da unidade $\mathrm{B}$ que, com autonomia plena, realizavam total acompanhamento dos pacientes com tuberculose, demonstrando reconhecimento diante da equipe de saúde do seu papel nesse cuidado.

\section{Conclusões}

Tendo em vista os aspectos observados neste estudo, conclui-se que a estrutura física adequada na farmácia das unidades de saúde, a disponibilidade de recursos materiais e humanos, com autonomia do profissional farmacêutico para o cuidado ao paciente com tuberculose, estabelecida por intermédio de uma boa relação com os profissionais envolvidos nesse cuidado, favorecem a consolidação e o desenvolvimento dos serviços farmacêuticos. Portanto, por meio de serviços farmacêuticos consolidados, é possível promover o efetivo cuidado ao paciente com tuberculose, na utilização adequada dos medicamentos, estabelecendo-se o fortalecimento da relação farmacêutico-paciente e consequente ampliação da adesão terapêutica.

Considerando-se os resultados obtidos, recomenda-se a ampliação e melhor organização dos serviços farmacêuticos para o cuidado ao paciente com tuberculose na rede de atenção primária do município de Salvador, tendo em vista que é possível desenvolver efetivamente esses serviços, como verificado em uma das unidades estudadas. Essa unidade pode servir como referência para as demais unidades da rede, mediante apoio da Coordenação Municipal de Assistência Farmacêutica.

Como limitações do estudo, destaca-se que a análise do processo de trabalho não foi contemplada no roteiro de observação sistemática. No entanto, os dados obtidos nas entrevistas realizadas com os farmacêuticos foram confrontados com aqueles obtidos nas entrevistas dos gerentes e usuários, contemplando assim esta análise.

Este estudo evidenciou a importância do farmacêutico no cuidado ao paciente com tuberculose. Assim, seus resultados podem contribuir para organização da AF na APS ao estabelecer critérios e padrões para avaliação dos serviços farmacêuticos no SUS. Ressaltase como potencialidade o instrumento elaborado para nortear a avaliação, o qual poderá servir como modelo para a gestão e para a 
assistência, bem como para novos estudos de avaliação na área de AF na APS.

\section{Colaboradores}

Rodrigues FF e Aquino R foram responsáveis pela concepção, planejamento, análise, interpretação dos dados, elaboração do rascunho, revisão crítica do conteúdo e aprovação da versão final do manuscrito. Medina MG foi responsável pela elaboração do rascunho, revisão crítica do conteúdo e aprovação da versão final do manuscrito.

\section{Referências}

1. Brasil. Ministério da Saúde. Secretaria de Vigilância em Saúde. Departamento de Vigilância Epidemiológica. Manual de recomendações para o controle da tuberculose no Brasil [internet]. Brasília, DF: MS; 2011 [acesso em 2014 mar 21]. Disponível em: http://bvsms.saude.gov.br/bvs/publicacoes/manual_recomendacoes_controle_tuberculose_brasil.pdf.

2. Pereira NC, Luiza VL, Cruz MM. Serviços farmacêuticos na atenção primária no município do Rio de Janeiro: um estudo de avaliabilidade. Saúde debate. 2015 abr-jun; 39(105):451-468.

3. Barreto JL, Guimarães MCL. Avaliação da gestão descentralizada da assistência farmacêutica básica em municípios baianos, Brasil. Cad Saúde Pública. 2010 jun; 26(6):1207-1220.

4. Oliveira LCF, Assis MMA, Barboni AR. Assistência Farmacêutica no Sistema Único de Saúde: da Política Nacional de Medicamentos à Atenção Básica à Saúde. Ciênc Saúde Colet. 2010; 15(supl.3):3561-3567.

5. Vieira FS. Assistência farmacêutica no sistema público de saúde no Brasil. Rev Panam Salud Pública. 2010; 27(2):149-156.
6. Araújo ALA, Pereira LRL, Ueta JM, et al. Perfil da assistência farmacêutica na atenção primária do Sistema Único de Saúde. Ciênc Saúde Colet. 2008; 13(supl):611617.

7. Leite SN, Manzini F, Álvares J, et. al. Infraestrutura das farmácias da atenção básica no Sistema Único de Saúde: Análise dos dados da PNAUM-Serviços. Rev Saúde Pública. 2017; 51(supl):2:13.

8. Brasil. Ministério da Saúde. Secretaria de Ciência, Tecnologia e Insumos Estratégicos. Departamento de Assistência Farmacêutica e Insumos Estratégicos. Diretrizes para estruturação de farmácias no âmbito do Sistema Único de Saúde. Série A. Normas e Manuais Técnicos [internet]. Brasília, DF: MS; 2009 [acesso em 2014 mar 21]. Disponível em: https://www.paho.org/bra/index.php?option=com docman\&view $=$ download $\&$ alias $=435$-diretrizes-para-estruturacao-farmacias-no-ambito-do-sistema-unico-saude-5\&category_slug=assistencia-farmaceutica-958\&Itemid=965.

9. Alencar TOS, Nascimento MAA, Alencar BR. Assistência Farmacêutica no SUS: articulando sujeitos, saberes e práticas. Feira de Santana: UEFS; 2011. 
10. Brasil. Ministério da Saúde. Secretaria de Ciência, Tecnologia e Insumos Estratégicos. Departamento de Assistência Farmacêutica e Insumos Estratégicos. Planejar é preciso: uma proposta para aplicação à Assistência Farmacêutica. 1. ed. Série B. Textos básicos de saúde [internet]. Brasília, DF: MS; 2006 [acesso em 2013 maio 25]. Disponível em: http://bvsms. saude.gov.br/bvs/publicacoes/06_1143_M.pdf.

11. Correia ARF, Mota DM, Arrais PSD, et.al. Definição de Indicadores para Avaliação da Assistência Farmacêutica na Rede Pública de Fortaleza-Ceará (Brasil) baseada em Métodos de Consenso. Latim American J Pharmacy. 2009; 28(3):366-374.

12. Souza LEPF, Vieira-da-Silva LM, Hartz ZMA. Conferencia de consenso sobre la imagen-objetivo de la descentralización de la atención de la salud en Brasil. In: Hartz ZMA, Vieira-da-Silva LM. Evaluación em Salud: de los modelos teóricos a La práctica em La evaluación de programas y sistemas de salud. 1. ed. Buenos Aires: Lugar Editorial; 2009.

13. Salvador. Secretaria municipal de saúde. Tabnet Salvador [internet]. Salvador: SMS; 2013 [acesso em 2013 abr 29]. Disponível em: http://www.tabnet.saude.salvador.ba.gov.br/deftohtm.exe?sinannet/notindivinet.def.

14. Organização Pan-Americana da Saúde, Organização Mundial da Saúde, Conselho Federal de Farmácia. O papel do farmacêutico no sistema de atenção à saúde. In: Relatório do Grupo Consultivo da OMS; $1988 \mathrm{dez}$ 13-16. Nova Délhi: OMS; 1988 [acesso em 2013 maio 20]. Disponível em: http://iris.paho.org/xmlui/bitstream/handle/123456789/3598/PapelFarmaceutico. pdf?sequence $=1$.

15. Organizacion Panamericana de la Salud. Servicios farmacéuticos basados en la atención primaria de salud. Documento de posición de la OPS/OMS. Washington: OPAS; 2013.
16. Alencar TOS, Nascimento MAA. Assistência Farmacêutica no Programa Saúde da Família: encontros e desencontros do processo de organização. Ciênc Saúde Colet. 2011; 16(9):3939-3949.

17. Moura BLA, Cunha RC, Fonseca ACF, et.al. Atenção primária à saúde: estrutura das unidades como componente da atenção à saúde. Rev Bras Saúde Matern Infant. 2010 nov; 10(supl.1):S69-S81.

18. Canabarro IM, Hahn S. Panorama da Assistência Farmacêutica na Saúde da Família em município do interior do Estado do Rio Grande do Sul. Epidemiol Serv Saúde. 2009 out-dez; 18(4):345-355.

19. Leite SN, Bernardo NLMC, Álvares J, et al. Serviço de dispensação de medicamentos na atenção básica no SUS. Rev Saúde Pública. 2017; 51(supl):2-11.

20. Castro MS, Chemello C, Pilger D, et al. Contribuição da atenção farmacêutica no tratamento de pacientes hipertensos. Rev Bras Hipertens. 2006; 13(3):198-202.

21. Nunes PHC, Pereira BMG, Nominato JCS, et al. Intervenção farmacêutica e prevenção de eventos adversos. Rev Bras Ciênc Farm. 2008 out-dez; 44(4):691699.

22. Ribeiro VF, Sapucaia KCG, Aragão LAO, et.al. Realização de intervenções farmacêuticas por meio de uma experiência em farmácia clínica. Rev Bras Farm Hosp Serv Saúde. 2015 out-dez; 6(4):18-22.

23. Araújo PS, Costa EA, Guerra Junior AA, et al. Atividades farmacêuticas de natureza clínica na atenção básica no Brasil. Rev Saúde Pública. 2017; 51(supl):2:6.

\footnotetext{
Recebido em 16/07/2017

Aprovado em 23/09/2018

Conflito de interesses: inexistente

Suporte financeiro: não houve
} 